\title{
A COMBINATORIAL INTERPRETATION OF THE LDU-DECOMPOSITION OF TOTALLY POSITIVE MATRICES AND THEIR INVERSES
}

\author{
Muhammad ElGebali and Nermine El-Sissi \\ The American University in Cairo, Egypt
}

\begin{abstract}
We study the combinatorial description of the LDUdecomposition of totally positive matrices. We give a description of the lower triangular $\mathrm{L}$, the diagonal $\mathrm{D}$, and the upper triangular $\mathrm{U}$ matrices of the LDU-decomposition of totally positive matrices in terms of the combinatorial structure of essential planar networks described by Fomin and Zelevinsky [5]. Similarly, we find a combinatorial description of the inverses of these matrices. In addition, we provide recursive formulae for computing the $\mathrm{L}, \mathrm{D}$, and $\mathrm{U}$ matrices of a totally positive matrix.
\end{abstract}

\section{INTRODUCTION}

The study of the class of totally positive matrices was initiated in the 1930s by F. R. Gantmacher and M. G. Krein ([6]). Also, an extensive study of totally positive matrices is covered in S. Karlin ([10]) and Fallat and Johnson ([4]). Totally positive matrices are a class of matrices that is worth investigating not only because of its mathematical beauty, but also because of their myriad of applications. More specifically, they arise in many applications, to name a few, statistics, approximation theory, operator theory, combinatorics, and planar resistor network $([5,8,10])$.

A matrix is said to be totally positive (totally nonnegative) if all its minors are positive (nonnegative). In this note, we focus our attention on totally positive matrices, but the results can be extended to nonsingular totally nonnegative matrices. Factorizations of totally positive and totally nonnegative,

2010 Mathematics Subject Classification. 15A23, 05C50.

Key words and phrases. Totally positive matrices, LDU factorization, planar networks. 
and totally nonpositive matrices have been examined in $[3,4,9]$ and [2], respectively, to minimize the computation of minors while testing for total positivity. More importantly, in [3, Lemma 5.1] and [7, Theorem 1] it was proved that

THEOREM 1.1. If a square matrix A has nonzero principal minors, then $A$ has a unique $L U$ factorization such that $L$ has a unit diagonal.

Since totally positive matrices have nonzero principal minors, the above Theorem provides a unique $L U$ factorization for totally positive matrices where $L$ is unit lower triangular. The Theorem also applies to invertible totally nonnegative matrices due to [1, Corollary 3.8]. In turn, this Theorem leads to a unique $L D U$ factorization where both $L$ and $U$ are lower and upper triangular matrices, respectively with unit diagonals and $D$ a diagonal matrix ([7, Theorem 2]).

In [5], the authors define an essential planar network, $\Gamma_{0}$ to be a directed (from left to right) planar network whose edges can either be slanted or horizontal in the middle of the network ([5, Figure 2$])$. All planar networks with $n$ sources and $n$ sinks are numbered bottom-to-top by $1,2, \ldots, n$, in [5]; whereas in this note, all planar networks are numbered bottom-to-top by $0,1, \ldots, n-1$; by the same token, all $n \times n$ matrices discussed here have rows and columns numbered from 0 to $n-1$. In section 2 , we give a detailed description of the planar network used in this work in light of the Definition of Fomin and Zelevinsky. This note adds to the combinatorial approach developed in [5] to study the parametrization of totally positive matrices.

It has been proved in [5, Theorem 5$]$ that $n \times n$ totally positive matrices are parametrized by $n^{2}$ positive parameters. More specifically, the parameters are the weights of an essential planar network and the entries of a totally positive matrix are recovered from these weights. In this note, we use these $n^{2}$ parameters to explicitly describe the entries of the matrices $L, D$, and $U$. Furthermore, we introduce recursive formulae for the $L, D$, and $U$ factors. Using these formulae, we compute the entries of an $n \times n$ matrix given an $(n-1) \times(n-1)$ matrix. More specifically, given $L D U$-factorization of an $(n-1) \times(n-1)$ totally positive matrix, we are able to compute the entries of the $L, D$ and $U$ of an $n \times n$ matrix. Also, we use the $n^{2}$ parameters to parametrize the inverse of a totally positive matrix.

In section 3, we obtain recursive formulae for computing the $(n+1) \times(n+1)$ lower, $L_{n}$, and upper triangular, $U_{n}$, matrices described in section 2 . We then provide closed-form formulae for the lower and upper triangular matrices in light of their corresponding planar subnetworks.

In section 4 , we provide a combinatorial description for computing the inverses of $L_{n}, D_{n}$, and $U_{n}$; and hence the inverse of a totally positive matrix. In addition, we obtain closed-form formulae for computing the entries of $L_{n}^{-1}$, $D_{n}^{-1}$ and $U_{n}^{-1}$. 
Finally, we present a numerical example in which we demonstrate the computation of the inverse of a totally positive matrix via concatenations of planar subnetworks. In addition, we provide a second example to recover the entries of a $4 \times 4$ totally positive upper triangular matrix via the recursive formula introduced in section 3 . Throughout this note, $\mathbb{N}$ denotes the set of natural numbers, and $\mathbb{N}_{0}$ the set of nonnegative integers.

\section{LDU-Decomposition of a Totally Positive Matrix}

In this section, we revisit Theorem 1.1 to set the stage for our results. More precisely, we use the fact that a totally positive matrix $A$ has a unique LDU-decomposition to describe three different subgraphs, representing the $L$, $D$, and $U$ matrices, respectively in terms of lattice paths to prove the main result of this section. More specifically, in our setting, we consider lattice paths of length $2 n+1$ on the first quadrant $\mathbb{N}_{0} \times \mathbb{N}_{0}$ which starts at $(0, i)$ and ends at $(2 n+1, j)$ to represent a path from source $i$ to sink $j$ on the essential planar network described in [5]. The only allowed steps are rise unit steps, horizontal unit steps, and fall unit steps. Throughout this note, the terms "edge" and "step" are used interchangeably.

We define a planar network of order $n$ to be an acyclic, planar directed graph. The vertices of this graph are points $(x, y) \in \mathbb{N}_{0} \times \mathbb{N}_{0}$ such that $x=$ $0,1, \ldots, 2 n+1$ and $y=0,1, \ldots, n$. The edges of this network are explained through subnetworks defined below. We assume that each network has $(n+$ $1)$-sources and $(n+1)$-sinks and all edges are directed from left to right, where each edge $\pi$ is assigned a scalar weight $\omega(\pi)$. In addition, the weight of a path $p$ from source $i$ to sink $j$ is defined as the product of the weights of its edges, namely $\omega(p)=\prod_{\pi \in p} \omega(\pi)$. The weight matrix of a planar network is the matrix whose $(i, j)$-entry is the sum of the weights of the paths from source $i$ to $\operatorname{sink} j$.

In our setting, we consider three planar subnetworks, which we call the $L$-type, the $D$-type and the $U$-type subnetworks. In this note, we do not distinguish between the terms "subnetworks" and "subgraphs."

The $L$-type subgraph of order $n$ is a planar network consisting of fall steps and horizontal steps.

Definition 2.1. For every $m, n \in \mathbb{N}$ define an $\mathcal{L}_{n}^{m}$-path to be any sequence of $n+1$ points, $\left(x_{k}, y_{k}\right) \in \mathbb{N}_{0} \times \mathbb{N}_{0}$ for $k=0,1,2, \ldots, n$ with the following conditions:

1. $x_{k}=m+k$ for all $k$.

2. $y_{k} \in\{0,1,2, \ldots, n\}$ for all $k$.

3. $y_{k+1} \in\left\{y_{k}, y_{k}-1\right\}$ for all $k<n$.

4. If $y_{k+1}=y_{k}-1$, then $y_{k} \geq n-k$.

Also, define $\mathcal{P}_{i, j}^{L_{n}}$ to be the set of all $\mathcal{L}_{n}^{0}$-paths with $y_{0}=i$ and $y_{n}=j$. 
In addition, the following Definition connects the $(n+1) \times(n+1)$ lower triangular matrix to Definition 2.1.

Definition 2.2. For every $n \in \mathbb{N}$ define $L_{n}$ to be the $(n+1) \times(n+1)$ matrix such that

$$
L_{n}[i, j]=\sum_{p \in \mathcal{P}_{i, j}^{L_{n}}} \omega_{L_{n}}(p)
$$

for all $0 \leq i, j \leq n$, where the weights of the edges are defined as follows,

- For $j \neq 0, \omega_{L_{n}}([(m+k, j),(m+k+1, j-1)])=t_{j, k+j-n}$,

- $\omega_{L_{n}}([(m+k, j),(m+k+1, j)])=1$,

for all $j, k=0,1, \ldots, n$.

Figure 1a shows an example of an $\mathcal{L}_{5}^{0}$-path. It consists of only horizontal and fall steps. Figure 1 b shows another path which is not an $\mathcal{L}_{5}^{0}$-path because the fall step from $(0,4)$ to $(1,3)$ is not admissible by the last condition in Definition 2.1.

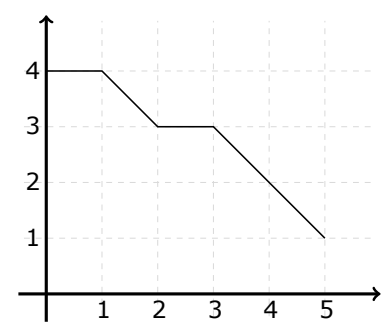

(a) An example of a path which is an $\mathcal{L}_{5}^{0}$-path

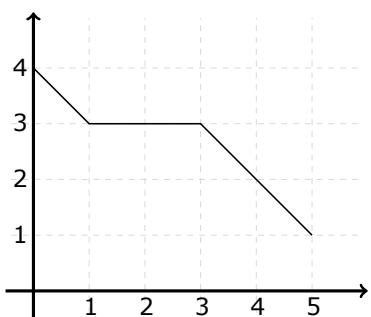

(b) An example of a path which is not an $\mathcal{L}_{5}^{0}$-path

FiguRE 1. Examples of lattice paths to illustrate Definition 2.1

The following Lemma and Corollary are a consequence of Definition 2.1.

LEMMA 2.3. If $\left[\left(x_{k}, y_{k}\right)\right]_{k=0, \ldots, n}$ is an $\mathcal{L}_{n}^{m}$-path, then $y_{k} \leq y_{l}$ whenever $k>l$.

The proof of the Lemma follows directly from the fact that $y_{k+1} \leq y_{k}$ for all $k<n$. The next Corollary shows that the matrix whose entries are defined in Definition 2.2 is unit lower triangular. The proof follows if we note that Lemma 2.3 implies that no path exists from source $i$ to sink $j$ if $i<j$, and exactly one path of weight 1 if $i=j$.

Corollary 2.4. For all $n \in \mathbb{N}$, the weight matrix of any L-type subnetwork of order $n$ is unit lower triangular. 


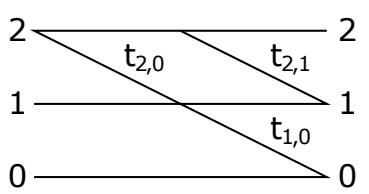

(a) $L$-type subnetwork of order 2 with weighting function $\omega_{L_{n}}$

$$
\left(\begin{array}{ccc}
1 & 0 & 0 \\
t_{1,0} & 1 & 0 \\
t_{2,0} t_{1,0} & t_{2,0}+t_{2,1} & 1
\end{array}\right)
$$

(b) The weight matrix $L_{2}$

Figure 2. Description of $L_{2}$

Figure 2 illustrates an $L$-type subnetwork of order 2 with weights defined as in Definition 2.2 and Figure $2 \mathrm{~b}$ is the weight matrix associated to it.

Similarly, we define another subgraph of order $n$, namely the $D$-type path. This path consists of horizontal steps only.

Definition 2.5. For every $m, n \in \mathbb{N}$, define a $\mathcal{D}_{n}^{m}$-path to be any sequence of the form $[(m, j),(m+1, j)]$ for some $j=0,1, \ldots, n$.

Also, define $\mathcal{P}_{i, j}^{D_{n}}$ to be the set of all $\mathcal{D}_{n}^{0}$-paths with $y_{0}=i$ and $y_{1}=j$.

In analogy to the $L$-type path, we can associate a matrix $D$ to a $D$-path.

Definition 2.6. For every $n \in \mathbb{N}$, define $D_{n}$ to be the $(n+1) \times(n+1)$ matrix such that

$$
D_{n}[i, j]=\sum_{p \in \mathcal{P}_{i, j}^{D_{n}}} \omega_{D_{n}}(p)
$$

for all $0 \leq i \leq n$; where $\omega_{D_{n}}[(m, i),(m+1, i)]=t_{i, i}$.

LEMma 2.7. For all $n \in \mathbb{N}, D_{n}$ is a diagonal matrix with diagonal elements $D_{n}[i, i]=t_{i, i}$. More specifically,

$$
D_{n}=\left(\begin{array}{cccc}
t_{0,0} & 0 & \cdots & 0 \\
0 & t_{1,1} & \cdots & 0 \\
\vdots & & \ddots & \vdots \\
0 & 0 & \cdots & t_{n, n}
\end{array}\right) .
$$

Proof. If $i \neq j$, then $D_{n}[i, j]=0$ from Definition 2.6; thus $D_{n}$ is a diagonal matrix. Now, for $i=j$, the set $\mathcal{P}_{i, i}^{D_{n}}=\{[(0, i),(1, i)]\}$. It follows that $D_{n}[i, i]=t_{i, i}$.

Finally, we define the $U$-type subnetwork in which only rise and horizontal steps are allowed.

Definition 2.8. For every $m, n \in \mathbb{N}$, define a $\mathcal{U}_{n}^{m}$-path to be any sequence of $n+1$ points $\left(x_{k}, y_{k}\right) \in \mathbb{N}_{0} \times \mathbb{N}_{0}$ for $k=0,1,2, \ldots, n$ with the following conditions:

1. $x_{k}=m+k$ for all $k$. 
2. $y_{k} \in\{0,1,2, \ldots, n\}$ for all $k$.

3. $y_{k+1} \in\left\{y_{k}, y_{k}+1\right\}$ for all $k<n$.

4. If $y_{k+1}=y_{k}+1$, then $y_{k} \geq k$.

Also, define $\mathcal{P}_{i, j}^{U_{n}}$ to be the set of all $\mathcal{U}_{n}^{0}$-paths with $y_{0}=i$ and $y_{n}=j$.

Then, we assign a matrix $U_{n}$ to the $U$-type subnetwork of order $n$.

Definition 2.9. For every $n \in \mathbb{N}$, define $U_{n}$ to be the $(n+1) \times(n+1)$ matrix such that

$$
U_{n}[i, j]=\sum_{p \in \mathcal{P}_{i, j}^{U_{n}}} \omega_{U_{n}}(p)
$$

for all $0 \leq i, j \leq n$, where the weight of the edges are defined as follows:

- For $j \neq 0, \omega_{U_{n}}([(m+k, j),(m+k+1, j+1)])=t_{j-k, j+1}$,

- $\omega_{U_{n}}([(m+k, j),(m+k+1, j)])=1$,

for all $j, k=0,1, \ldots, n$.

Figure 3 shows an example of a $\mathcal{U}_{6}^{0}$-path. It consists of only horizontal and rise steps.

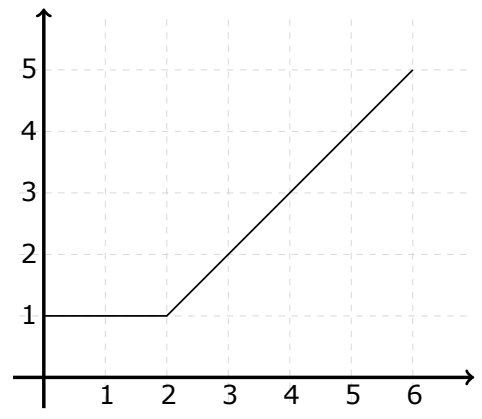

FiguRE 3. A $\mathcal{U}_{6}^{0}$-path

The proofs of the following Lemma and Corollary are similar to Lemma 2.3 and Corollary 2.4 .

LEMMA 2.10. If $\left[\left(x_{k}, y_{k}\right)\right]_{k=0, \ldots, n}$ is a $\mathcal{U}_{n}^{m}$-path, then $y_{k} \geq y_{l}$ whenever $k>l$.

COROLlary 2.11. For all $n \in \mathbb{N}$, the weight matrix of a $U$-type subnetwork of order $n$ is unit upper triangular.

In our context, we define an essential planar network of order $n$ to be the (ordered) concatenation of an $L$-type, a $D$-type and a $U$-type subnetworks of order $n$. Equivalently, a path in our network is the concatenation of an $\mathcal{L}_{n}^{0}$, a $\mathcal{D}_{n}^{n}$ and a $\mathcal{U}_{n}^{n+1}$ paths, respectively. From this Definition it is clear 
that our network is an acyclic directed (from left to right) planar graph. The restrictions on the fall and rise steps given in Definitions 2.2 and 2.9 guarantee that our slanted edges match the essential edges of the planar network defined in [5]. This implies that our Definition of the essential planar network is equivalent to the Definition of Fomin and Zelevinsky.

In this section, we constructed an $L_{n}$ lower triangular matrix obtained from an $L$-type subnetwork of order $n$, and similarly, the $D$-type and $U$-type subnetworks were used to recover the entries of a diagonal matrix $D_{n}$ and an upper triangular matrix $U_{n}$, respectively. In addition, we use the fact that the concatenation of these networks is equivalent to computing the product of their corresponding weight matrices $([4,5])$ to obtain the main Theorem of this section.

TheOREM 2.12. The LDU decomposition of a totally positive matrix $A$ can be recovered by decomposing the essential planar network associated with $A$ into an L-type, a D-type and a $U$-type subnetworks, respectively.

The proof of this Theorem follows directly from [5, Theorem 5], Corollaries 2.4, 2.11, Lemma 2.7 and the fact that the weight matrix corresponding to the concatenated planar networks is the product of the weight matrices of the original network $([4,5])$.

Figure 4 illustrates an example of an essential planar network of order 2 obtained by concatenating three essential planar subnetworks of order 2 .
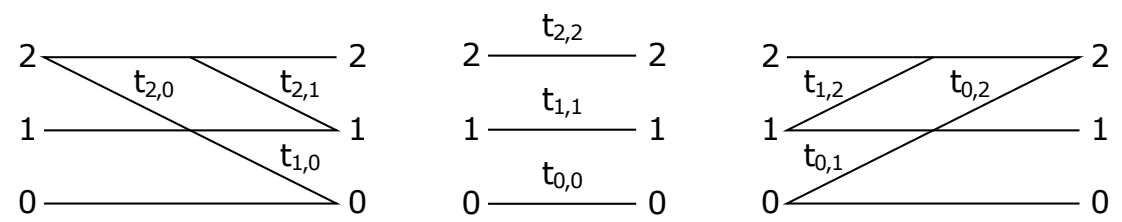

(a) $L$-type subnetwork of (b) $D$-type subnetwork of (c) $U$-type subnetwork of order 2 order 2 order 2

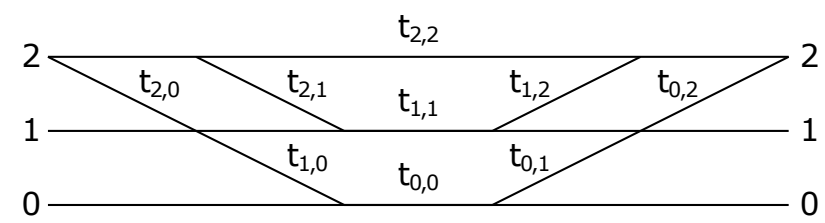

(d) Essential planar network of order 2

Figure 4. Concatenation of essential planar subnetworks of order 2 to produce the essential planar network of order 2 


\section{FormulaE FOR $L_{n}$ AND $U_{n}$}

In this section we describe how we can compute the entries of the lower triangular matrix $L_{n}$ and the upper triangular matrix $U_{n}$ presented in the previous section. We begin with a recursive formula for $L_{n}$. To clarify the notation, $I_{n}$ is the $n \times n$ identity matrix, $\mathbf{0}_{n}$ is the column vector of $n$ zeros, and $L_{n}^{-1}$ is the inverse of $L_{n}$, whose existence and complete description is presented in the following section.

Proposition 3.1. If $L_{n}$ is defined as in Definition 2.2, then $L_{n+1}$ can be computed recursively in the following way,

- $L_{0}=(1)$,

- $L_{n+1}=F_{L_{n}}\left(L_{n} \oplus 1\right)$, where $F_{L_{n}}=\left(\begin{array}{cc}I_{n+1} & \mathbf{0}_{n+1} \\ -L_{n+1}^{-1}[n+1, j]_{0 \leq j \leq n} & 1\end{array}\right)$.

Proof. We use induction to prove the Proposition. The base case is trivial. We consider the inductive step, $L_{n+1}=F_{L_{n}}\left(L_{n} \oplus 1\right)$. We begin by expanding the product $F_{L_{n}}\left(L_{n} \oplus 1\right)$ as follows:

$$
\begin{aligned}
F_{L_{n}}\left(L_{n} \oplus 1\right) & =\left(\begin{array}{cc}
I_{n+1} & \mathbf{0}_{n+1} \\
-L_{n+1}^{-1}[n+1, j]_{0 \leq j \leq n} & 1
\end{array}\right)\left(\begin{array}{cc}
L_{n} & \mathbf{0}_{n+1} \\
\mathbf{0}_{n+1}^{\top} & 1
\end{array}\right) \\
& =\left(\begin{array}{cc}
L_{n} & \mathbf{0}_{n+1} \\
-L_{n+1}^{-1}[n+1, j]_{0 \leq j \leq n} L_{n} & 1
\end{array}\right) .
\end{aligned}
$$

First, we show that $L_{n+1}[i, j]_{0 \leq i, j \leq n}=L_{n}$. This can be best described combinatorially, where a path $p \in \mathcal{P}_{i, j}^{L_{n+1}}(i \leq n)$ is equivalent to a horizontal step $[(0, i),(1, i)]$ followed by an $\mathcal{L}_{n}^{1}$-path $p^{\prime}$. Notice that a fall step $[(0, i),(1, i-1)]$ is not allowed since $i<n+1$ (see Definition 2.1). It follows that $\omega_{L_{n+1}}(p)=\omega_{L_{n}}\left(p^{\prime}\right)$, and our description clearly shows a bijection. So, indeed $L_{n+1}[i, j]=L_{n}[i, j]$ for $i, j \in\{0, \ldots, n\}$.

The rightmost column is trivial since $L_{n+1}$ is unit lower triangular by Corollary 2.4. Finally, we need to show that

$$
L_{n+1}[n+1, j]_{0 \leq j \leq n}=-L_{n+1}^{-1}[n+1, j]_{0 \leq j \leq n} L_{n} .
$$

We begin by the simple fact that

$$
L_{n+1}^{-1} L_{n+1}=I_{n+1} .
$$

Now, we rewrite it in block matrix notation, where $L_{n+1}^{-1}[i, j]_{0 \leq i, j \leq n}=L_{n}^{-1}$ to guarantee the product is the identity matrix.

$$
\begin{aligned}
& \left(\begin{array}{cc}
L_{n}^{-1} & \mathbf{0}_{n+1} \\
L_{n+1}^{-1}[n+1, j]_{0 \leq j \leq n} & 1
\end{array}\right)\left(\begin{array}{cc}
L_{n} & \mathbf{0}_{n+1} \\
L_{n+1}[n+1, j]_{0 \leq j \leq n} & 1
\end{array}\right) \\
& =\left(\begin{array}{cc}
I_{n+1} & \mathbf{0}_{n+1} \\
\mathbf{0}_{n+1}^{\top} & 1
\end{array}\right) .
\end{aligned}
$$


Then, consider the product of the second row with the first column which produce the following equation

$$
L_{n+1}^{-1}[n+1, j]_{0 \leq j \leq n} L_{n}+L_{n+1}[n+1, j]_{0 \leq j \leq n}=\mathbf{0}_{n+1}^{\top}
$$

which, if rearranged, will show that

$$
L_{n+1}[n+1, j]_{0 \leq j \leq n}=-L_{n+1}^{-1}[n+1, j]_{0 \leq j \leq n} L_{n} .
$$

In a similar fashion, we define a recursive formula for $U_{n}$ as follows:

Proposition 3.2. If $U_{n}$ is defined as in Definition 2.9, then it follows the following recursion

- $U_{0}=(1)$,

- $U_{n+1}=\left(U_{n} \oplus 1\right) F_{U_{n}}$, where $F_{U_{n}}=\left(\begin{array}{cc}I_{n+1} & -U_{n+1}^{-1}[i, n+1]_{0 \leq i \leq n} \\ \mathbf{0}_{n+1}^{\top} & 1\end{array}\right)$.

Since the proof follows the same ideas as Proposition 3.1, it will be omitted.

The following Lemma sets the stage for the main result of this section. More specifically, we provide a closed-form formula to compute the entries of $L_{n}$ ane $U_{n}$, respectively.

Lemma 3.3. For all $n \in \mathbb{N}$ and $0 \leq i, j \leq n$, let $p \in \mathcal{P}_{i, j}^{L_{n}}$ and $\pi_{1}, \pi_{2} \in p$ such that $\omega_{L_{n}}\left(\pi_{1}\right)=t_{y_{1}, s_{1}}$ and $\omega_{L_{n}}\left(\pi_{2}\right)=t_{y_{2}, s_{2}}$ where $\omega_{L_{n}}$ is defined as in Definition 2.2. If $y_{1}<y_{2}$, then $s_{1} \geq s_{2}$.

Proof. From Definition 2.2, we conclude that there are $x_{1}, x_{2} \in$ $\{0, \ldots, n-1\}$ such that $\pi_{1}=\left[\left(m+x_{1}, y_{1}\right),\left(m+x_{1}+1, y_{1}-1\right)\right]$ and $\pi_{2}=\left[\left(m+x_{2}, y_{2}\right),\left(m+x_{2}+1, y_{2}-1\right)\right]$. It follows from the same Definition that $s_{1}=x_{1}+y_{1}-n$ and $s_{2}=x_{2}+y_{2}-n$. Consequently, $s_{1}-s_{2}=\left(x_{1}-x_{2}\right)+\left(y_{1}-y_{2}\right)$. Given that $y_{1}<y_{2}$ and from Lemma 2.3, $x_{1}>x_{2}$, which means $x_{1}-x_{2}>0$ and $y_{1}-y_{2}<0$. Nevertheless, we know that each step $x$ increases by 1 while $y$ decreases by 1 or remains the same. It is obvious then that $x_{1}-x_{2} \geq-\left(y_{1}-y_{2}\right)$. In other words, $s_{1}-s_{2} \geq 0$.

In the following Theorem, we define $Q_{i, j}^{I}$, for $i>j$, to be the set of increasing sequences of length $i-j$. Let $\alpha$ be a sequence in $Q_{i, j}^{I}$, then $0 \leq$ $\alpha_{r} \leq i-r$, where $\alpha_{r}$ is the $r$-th element of the sequence $\alpha$. For $i<j$, we define $Q_{i, i}^{I}=\{\varepsilon\}$, and $Q_{i, j}^{I}=\phi$ where $\varepsilon$ is the empty sequence.

Theorem 3.4. If $L_{n}$ is defined as in Definition 2.2, then

$$
L_{n}[i, j]=\sum_{\alpha \in Q_{i, j}^{I}} \prod_{r=j}^{i-1} t_{r+1, \alpha_{i-r}} .
$$


Proof. If $i<j$, then $Q_{i, j}^{I}=\phi$. Thus, the sum is empty and $L_{n}[i, j]=0$. If $i=j$, the sum is over the empty sequence $\varepsilon$ only. It follows that

$$
L_{n}[i, j]=\prod_{r=j}^{i-1} t_{r+1, \varepsilon_{i-r}}=1
$$

since it is an empty product. These two results agree with Corollary 2.4. Finally, we need to show that (3.1) holds for the case $i>j$. The proof relies on constructing a bijection between $Q_{i, j}^{I}$ and $\mathcal{P}_{i, j}^{L_{n}}$ such that if $\alpha \mapsto p$, then $\omega_{L_{n}}(p)=\prod_{r=j}^{i-1} t_{r+1, \alpha_{i-r}}$. Define $f(\alpha)=\left[\left(k, y_{k}\right)\right]_{0 \leq k \leq n}$ such that

$$
y_{k}=\left\{\begin{array}{cl}
i, & \text { if } 0 \leq k \leq \alpha_{1}+n-i \\
i-r, & \text { if } \alpha_{r}+n-(i-r) \leq k \leq \alpha_{r+1}+n-(i-r) \\
& \text { for } r \in\{1, \ldots, i-j-1\} \\
j, & \text { if } \alpha_{i-j}+n-j \leq k \leq n
\end{array} .\right.
$$

First, we show $f$ is well defined. By Definition of $Q_{i, j}^{I}$, we know that $0 \leq \alpha_{1} \leq$ $i-1$ and $\alpha_{r} \leq \alpha_{r+1} \leq i-(r+1)$. It follows that $0 \leq n-i \leq \alpha_{1}+n-i \leq n-1$ and $\alpha_{r}+n-(i-r) \leq \alpha_{r+1}+n-(i-r) \leq n-1$ for $r \in\{1, \ldots, i-j-1\}$. By letting $r=i-j-1$, we get $\alpha_{i-j}+n-j \leq n$. Combining these inequalities yields

$$
\begin{aligned}
0 & \leq \alpha_{1}+n-i<\alpha_{1}+n-(i-1) \leq \alpha_{2}+n-(i-1) \\
& <\alpha_{2}+n-(i-2) \cdots \leq \alpha_{i-j}+n-(j+1)<\alpha_{i-j}+n-j \leq n,
\end{aligned}
$$

which shows that by fixing $k$ there is a unique $y_{k}$. It also proves that $y_{0}=i$, $y_{n}=j$, and if $k_{1}<k_{2}$, then $y_{k_{1}} \geq y_{k_{2}}$. It is easy to see from these that if $y_{k}=s$ for $s \in\{j+1, \ldots, i\}$, then $y_{k+1} \in\{s, s-1\}$. It remains to show that if $y_{k+1}=y_{k}-1$, then $y_{k} \geq n-k$. Assume $y_{k+1}=s$ for $s \in\{j, \ldots, i-1\}$. From the Definition, we know that $k+1=\alpha_{i-s}+n-s$. Therefore, $n-k=\alpha_{i-s}+s+1$. However, $y_{k}=s+1$ and $\alpha_{i-s} \geq 0$. As a consequence, $y_{k} \geq n-k$. Now, we can conclude that $f(\alpha) \in \mathcal{P}_{i, j}^{L_{n}}$.

To show that $f$ is surjective, assume $p \in \mathcal{P}_{i, j}^{L_{n}}$ and $p=\left[\left(k, y_{k}\right)\right]$. Define $K$ to be the set of indices $k$ at which there is a fall step. More precisely, $K=\left\{k: k<n\right.$ and $\left.y_{k+1}=y_{k}-1\right\}$. Clearly, $K$ has $i-j$ elements, so call them $k_{1}, \ldots, k_{i-j}$ in ascending order. Now, define $\beta$ such that,

$$
\beta_{r}=k_{r}+i-r+1-n .
$$

$k_{r}$ is strictly increasing, so $k_{r}-r$ is increasing. It follows that $\beta_{r}$ is increasing. Since $k_{r}<n, k_{r}+1-n \leq 0$, and, consequently, $\beta_{r} \leq i-r$. Finally, we know from condition (iii) in Definition 2.1 that $k_{r} \geq n-y_{k_{r}}$. Also, by Definition, $y_{k_{1}}=i$ and $y_{k_{r+1}}=y_{k_{r}}-1$, which imply that $y_{k_{r}}=i-(r-1)$. This implies that $k_{r} \geq n-i+r-1$, which implies directly that $\beta_{r} \geq 0$. To sum up the results, we showed that $\beta$ is increasing and $0 \leq \beta_{r} \leq i-r$. Therefore, $\beta \in Q_{i, j}^{I}$ and $f(\beta)$ is $p$. 
To show that $f$ is injective, assume two distinct sequences $\alpha, \beta \in Q_{i, j}^{I}$. Define $D$ to be the set of indices at which they differ. More precisely, $D=$ $\left\{1 \leq r \leq i-j: \alpha_{r} \neq \beta_{r}\right\}$ which is clearly non-empty. Take $r_{o}$ to be the minimum of $D$, and assume $f(\alpha)=\left[\left(k, y_{k}^{\alpha}\right)\right]$ and $f(\beta)=\left[\left(k, y_{k}^{\beta}\right)\right]$. Since $\alpha_{r_{o}} \neq \beta_{r_{o}}$, assume, without loss of generality, that $\alpha_{r_{o}}<\beta_{r_{o}}$. Let $k_{c}$ be $\alpha_{r_{o}}+n-\left(i-r_{o}\right)$, so $k_{c}<\beta_{r_{o}}+n-\left(i-r_{o}\right)$. Therefore, $y_{k_{c}}^{\alpha}=i-r_{o}$ while $y_{k_{c}}^{\beta}=i-r_{o}-1$. Consequently, $f(\alpha) \neq f(\beta)$, and hence the injectivity of $f$.

Therefore, $f$ is a bijection between $Q_{i, j}^{I}$ and $\mathcal{P}_{i, j}^{L_{n}}$. It remains to show that

$$
\omega_{L_{n}}(f(\alpha))=\prod_{r=j}^{i-1} t_{r+1, \alpha_{i-r}} .
$$

Once more, consider the set $K=\left\{k: k<n\right.$ and $\left.y_{k+1}=y_{k}-1\right\}$. Let $\pi_{k}$ be the edge $\left[\left(k, y_{k}\right),\left(k+1, y_{k+1}\right)\right]$ in $f(\alpha)$, for a fixed $0 \leq k<n$. It is clear from Definition 2.2 that

$$
\omega_{L_{n}}\left(\pi_{k}\right)=\left\{\begin{array}{cl}
t_{y_{k}, k+y_{k}-n}, & \text { if } k \in K \\
1, & \text { if } k \notin K
\end{array},\right.
$$

since $\omega_{L_{n}}(f(\alpha))=\prod_{k=0}^{n-1} \omega_{L_{n}}\left(\pi_{k}\right)$, we deduce that

$$
\omega_{L_{n}}(f(\alpha))=\prod_{k \in K} t_{y_{k}, k+y_{k}-n} .
$$

Using the order described on $K$, we rewrite the last formula as

$$
\omega_{L_{n}}(f(\alpha))=\prod_{r=1}^{i-j} t_{y_{k_{r}}, k_{r}+y_{k_{r}}-n} .
$$

However, we have already proved that $y_{k_{r}}=i-(r-1)$. Therefore, if we substitute the value of $y_{k_{r}}$ and apply the transformation $s=i-r$, we reach the formula $\omega_{L_{n}}(f(\alpha))=\prod_{s=j}^{i-1} t_{s+1, k_{i-s}+s+1-n}$. Using (3.2), we know that $\alpha_{i-s}=k_{i-s}+i-((i-s)-1)-n=k_{i-s}+s+1-n$. Thus, substituting back in the last formula and replacing $s$ with $r$, we conclude that $\omega_{L_{n}}(f(\alpha))=$ $\prod_{r=j}^{i-1} t_{r+1, \alpha_{i-r}}$. Now, we use Definition 2.2, to write

$$
L_{n}=\sum_{p \in \mathcal{P}_{i, j}^{L_{n}}} \omega_{L_{n}}(p)=\sum_{\alpha \in Q_{i, j}^{I}} \prod_{r=j}^{i-1} t_{r+1, \alpha_{i-r}} .
$$

As the case of $L_{n}$, there is a closed-form formula for the entries of the matrix $U_{n}$ in terms of the $t_{i, j}$ parameters. This is highlighted in the following Theorem. 
Theorem 3.5. If $U_{n}$ is defined as in Definition 2.9, then

$$
U_{n}[i, j]=\sum_{\alpha \in Q_{j, i}^{I}} \prod_{r=i}^{j-1} t_{\alpha_{j-r}, r+1}
$$

Proof. If we let

$$
y_{k}=\left\{\begin{array}{cl}
j, & \text { if } j-\alpha_{1} \leq k \leq n \\
j-r, & \text { if } j-r-\alpha_{r+1} \leq k \leq j-r-\alpha_{r} \text { for } r \in\{1, \ldots, j-i-1\} \\
i, & \text { if } 0 \leq k \leq i-\alpha_{j-i}
\end{array}\right.
$$

then a similar argument to the one in the proof of Theorem 3.4 is used.

\section{The Inverse of a Totally Positive Matrix}

The Definition of totally positive matrices introduced in Section 1 ensures that this class of matrices is invertible. In Section 2, we used the fact that every totally positive matrix has a unique LDU-decomposition and provided a combinatorial description of the $L_{n}, D_{n}$, and $U_{n}$. If the inverses of the $L_{n}$, $D_{n}$ and $U_{n}$ factors are known to be $L_{n}^{-1}, D_{n}^{-1}$ and $U_{n}^{-1}$, respectively, then the inverse of the totally positive matrix can be computed as the product $U_{n}^{-1} D_{n}^{-1} L_{n}^{-1}$. Also, since the product of the $L_{n}, D_{n}$ and $U_{n}$ factors yields a matrix that is invertible, then each of the factors is also invertible. In this section, we provide a combinatorial description of the inverse of each of these factors, similar to the one described in Section 2.

A new weight matrix, which we call $L_{n}^{-1}$, is recovered from an $L$-type subnetwork whose weights are defined as follows:

Definition 4.1. For all $n \in \mathbb{N}_{0}$, define $\omega_{L_{n}^{-1}}$ such that:

- for $j \neq 0, \omega_{L^{-1}}([(m+k, j),(m+k+1, j-1)])=-t_{j, n-k-1}$,

- $\omega_{L_{n}^{-1}}([(m+k, j),(m+k+1, j)])=1$

for all $0 \leq k, j \leq n$.

It follows from Corollary 2.4 that $L_{n}^{-1}$ is unit lower triangular. In this section, a formula for the entries of $L_{n}^{-1}$ is obtained and we use it to show that $L_{n}^{-1}$ is indeed the inverse of $L_{n}$.

Lemma 4.2. For all $n \in \mathbb{N}$ and $0 \leq i, j \leq n$, let $p \in \mathcal{P}_{i, j}^{L_{n}}$ and $\pi_{1}, \pi_{2} \in p$ such that $\omega_{L_{n}^{-1}}\left(\pi_{1}\right)=-t_{y_{1}, s_{1}}$ and $\omega_{L_{n}^{-1}}\left(\pi_{2}\right)=-t_{y_{2}, s_{2}}$ where $\omega_{L_{n}^{-1}}$ is defined as in Definition 4.1. If $y_{1}<y_{2}$, then $s_{1}<s_{2}$.

Proof. From Definition 4.1, we conclude that there are $x_{1}, x_{2} \in$ $\{0, \ldots, n-1\}$ such that $\pi_{1}=\left[\left(m+x_{1}, y_{1}\right),\left(m+x_{1}+1, y_{1}-1\right)\right]$ and $\pi_{2}=\left[\left(m+x_{2}, y_{2}\right),\left(m+x_{2}+1, y_{2}-1\right)\right]$. It follows from the same Definition that $s_{1}=n-x_{1}-1$ and $s_{2}=n-x_{2}-1$. Consequently, $s_{1}-s_{2}=x_{2}-x_{1}$. Given that $y_{1}<y_{2}$ and from Lemma 2.3, $x_{1}>x_{2}$, which means $x_{2}-x_{1}<0$. Therefore, $s_{1}-s_{2}<0$. 
In analogy to Theorem 3.4, we give a closed-form formula for $L_{n}^{-1}$. To prove this, define $Q_{i, j}^{S D}$, for $i>j$, to be the set of strictly decreasing sequences of length $i-j$, and if $\alpha \in Q_{i, j}^{S D}$, then $0 \leq \alpha_{r} \leq i-r$, where $\alpha_{r}$ is the $r$-th element of the sequence $\alpha$. Also, we extend the Definition such that $Q_{i, i}^{S D}=\{\varepsilon\}$ for $i=j$ and $Q_{i, j}^{S D}=\phi$, the empty set for $i<j$.

THEOREM 4.3. If $L_{n}^{-1}$ is the weight matrix of the L-type subnetwork of order $n$ whose weights are defined as in Definition 4.1, then

$$
L_{n}^{-1}[i, j]=(-1)^{i-j} \sum_{\alpha \in Q_{i, j}^{S D}} \prod_{r=j}^{i-1} t_{r+1, \alpha_{i-r}}
$$

Proof. If we let

$$
y_{k}=\left\{\begin{array}{cl}
i, & \text { if } 0 \leq k \leq n-\alpha_{1}-1 \\
i-r, & \text { if } n-\alpha_{r} \leq k \leq n-\alpha_{r+1}-1 \text { for } r \in\{1, \ldots, i-j-1\} \\
j, & \text { if } n-\alpha_{i-j} \leq k \leq n
\end{array}\right.
$$

then a similar argument to the one in the proof of Theorem 3.4 is used.

We observe that $L_{n}^{-1}$ shares quite similar characteristics as the ones of the lower triangular matrix $L_{n}$ presented in Section 2. It is not surprising to see that $L_{n}^{-1}$, the way defined, is the inverse of $L_{n}$ defined in Definition 2.2.

THEOREM 4.4. If $L_{n}$ and $L_{n}^{-1}$ are the weight matrices of the L-type subnetwork of order $n$ whose weights are defined as in Definitions 2.2 and 4.1, respectively, then $L_{n} L_{n}^{-1}=I_{n+1}$, the $(n+1) \times(n+1)$ identity matrix.

Proof. From Corollary 2.4, we know that both $L_{n}$ and $L_{n}^{-1}$ are unit lower triangular, and thus, their product is unit lower triangular as well. It remains to show that the dot product of row $i$ of $L_{n}$ with column $j$ of $L_{n}^{-1}$ is 0 , whenever $i>j$. To show that, we let $A_{n}=L_{n} L_{n}^{-1}$ and expand the product as

$$
A_{n}[i, j]=\sum_{k=0}^{n} L_{n}[i, k] L_{n}^{-1}[k, j]
$$

which can be simplified, using Theorems 3.4 and 4.3, as

$$
A_{n}[i, j]=\sum_{k=j}^{i} T_{k}
$$

where

$$
T_{k}=(-1)^{k-j} \sum_{\alpha \in Q_{i, k}^{I}} \sum_{\beta \in Q_{k, j}^{S D}} \prod_{r \in\{k \ldots i-1\}} t_{r, \alpha_{i-r}} \prod_{s \in\{j \ldots k-1\}} t_{s, \beta_{k-s}} .
$$


Here we observe that if $k<j\left(\right.$ or $k>i$ ), then $L_{n}^{-1}[k, j]=0$ (or $L_{n}[i, k]=$ 0 ) by Lemma 2.4. Then, we define two more sums, $T_{k}^{\leq}$and $T_{k}^{>}$as follows

$$
\begin{aligned}
& T_{k}^{\leq}= \\
& \left\{\begin{array}{cll}
(-1)^{k-j} \sum_{\alpha \in Q_{i, k}^{I}} \sum_{\substack{\beta \in Q_{k, j}^{S D} \\
\alpha_{i-k} \leq \beta_{1}}} \prod_{r \in\{k \ldots i-1\}} t_{r, \alpha_{i-r}} & \text { if } j<k<i \\
T_{k}, & \prod_{s \in\{j \ldots k-1\}} t_{s, \beta_{k-s},}, & \text { if } k=i \\
0, & \text { if } k=j
\end{array},\right.
\end{aligned}
$$

$$
\begin{aligned}
& T_{k}^{>}= \\
& \left\{\begin{array}{rll}
(-1)^{k-j} \sum_{\alpha \in Q_{i, k}^{I}} \sum_{\substack{\beta \in Q_{k, j}^{S D} \\
\alpha_{i-k}>\beta_{1}}} \prod_{r \in\{k \ldots i-1\}} t_{r, \alpha_{i-r}} & \text { if } j<k<i \\
0, & \prod_{s \in\{j \ldots k-1\}} t_{s, \beta_{k-s},}, & \text { if } k=i \\
T_{k}, & \text { if } k=j
\end{array} .\right.
\end{aligned}
$$

Clearly, $T_{k}=T_{k}^{\leq}+T_{k}^{>}$, where $T_{k}^{\leq}$and $T_{k}^{>}$have no common terms. It follows from (4.2a) that $A_{n}[i, j]=\sum_{k=j}^{i}\left(T_{k}^{\leq}+T_{k}^{>}\right)$. Rearranging the terms, the sum becomes

$$
A_{n}[i, j]=T_{j}^{\leq}+\sum_{k=j}^{i-1}\left(T_{k}^{>}+T_{k+1}^{\leq}\right)+T_{i}^{>}
$$

From Definition (4.2), $T_{j}^{\leq}=T_{i}^{>}=0$. Thus, $A_{n}[i, j]=\sum_{k=j}^{i-1}\left(T_{k}^{>}+T_{k+1}^{\leq}\right)$. We will now show that $T_{k}^{>}=-T_{k+1}^{\leq}$for all $j \leq k<i$ so that $A_{n}[i, j]=0$ as desired.

Since $\alpha_{i-k}>\beta_{1}$, define new sequences $\alpha^{\prime}$ and $\beta^{\prime}$ such that $\alpha_{r}^{\prime}=\alpha_{r}$ for $r=1, \ldots, i-k-1, \beta_{1}^{\prime}=\alpha_{i-k}$, and $\beta_{r}^{\prime}=\beta_{r-1}$ for $r=2, \ldots, k+1-j$. Since $\alpha$ is increasing, $\alpha_{i-k-1}^{\prime} \leq \beta_{1}^{\prime}$. Therefore, $T_{k}^{>}$can be rewritten as

$$
T_{k}^{>}=(-1)^{k-j} \sum_{\alpha^{\prime} \in Q_{i, k+1}^{I}} \sum_{\substack{\beta^{\prime} \in Q_{k+1, j}^{S D} \\ \alpha_{i-k-1}^{\prime} \leq \beta_{1}^{\prime}}} \prod_{r \in\{k+1, \ldots, i-1\}} t_{r, \alpha_{i-r}^{\prime}} \prod_{s \in\{j \ldots k\}} t_{s, \beta_{k+1-s}^{\prime}},
$$

which differs from $T_{k+1}^{\leq}$by a factor of -1 . Therefore, $A_{n}=I_{n+1}$. 
Then, similar to the case of $L_{2}$, we can now present an example (in Figure 5) of an $L$-type subnetwork of order 2 with weights defined as in Definition 4.1, and the weight matrix of that network, namely $L_{2}^{-1}$.

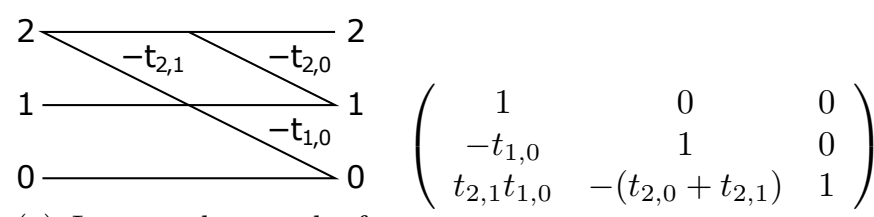

(a) $L$-type subnetwork of order 2 with weighting function $\omega_{L_{n}^{-1}}$

(b) The weight matrix $L_{2}^{-1}$

FiguRE 5. Description of $L_{2}^{-1}$

Similarly, we define the inverse of $D_{n}$. All the entries on the diagonal of $D_{n}$ are positive, so it is invertible. The inverse, namely $D_{n}^{-1}$ is the diagonal matrix whose diagonal entries are the reciprocals of the diagonal elements of $D_{n}$. Combinatorially, $D_{n}^{-1}$ can be recovered from a $D$-type subnetwork of order $n$ where the weights are defined as

$$
\omega_{D_{n}^{-1}}([(0, i),(1, i)])=\frac{1}{t_{i, i}},
$$

for $i=0, \ldots, n$. In analogy to Lemma 2.7 , the general form of $D_{n}^{-1}$ is

$$
D_{n}^{-1}=\left(\begin{array}{cccc}
\frac{1}{t_{0,0}} & 0 & \cdots & 0 \\
0 & \frac{1}{t_{1,1}} & \cdots & 0 \\
\vdots & & \ddots & \vdots \\
0 & 0 & \cdots & \frac{1}{t_{n, n}}
\end{array}\right) .
$$

To find the inverse of $U_{n}$, we will mimic the same approach employed to define $L_{n}^{-1}$. We define $U_{n}^{-1}$ to be the weight matrix of a $U$-type subnetwork whose weights are given in the following Definition.

Definition 4.5. For all $n \in \mathbb{N}_{0}$, define $\omega_{U_{n}^{-1}}$ such that:

- for $j \neq 0, \omega_{U_{n}^{-1}}([(m+k, j),(m+k+1, j-1)])=-t_{k, j}$,

- $\omega_{U_{n}^{-1}}([(m+k, j),(m+k+1, j)])=1$,

for all $0 \leq k, j \leq n$.

It follows from Corollary 2.11 that $U_{n}^{-1}$ is unit upper triangular. Consequently, we obtain the following Theorem. 
THEOREM 4.6. If $U_{n}^{-1}$ is the weight matrix of the $U$-type subnetwork of order $n$ whose weights are defined as in Definition 4.5, then

$$
U_{n}^{-1}[i, j]=(-1)^{i-j} \sum_{\alpha \in Q_{j, i}^{S D}} \prod_{r=i}^{j-1} t_{\alpha_{j-r}, r+1}
$$

Proof. If we let

$$
y_{k}=\left\{\begin{array}{cl}
j, & \text { if } \alpha_{1}+1 \leq k \leq n \\
j-r, & \text { if } \alpha_{r+1}+1 \leq k \leq \alpha_{r} \text { for } r \in\{1, \ldots, j-i-1\} \\
i, & \text { if } 0 \leq k \leq \alpha_{j-i}
\end{array}\right.
$$

then a similar argument to the one in the proof of Theorem 3.4 is used.

THEOREM 4.7. If $U_{n}$ and $U_{n}^{-1}$ are the weight matrices of the $U$-type subnetwork of order $n$ whose weights are defined as in Definitions 2.9 and 4.5, respectively, then $U_{n} U_{n}^{-1}=I_{n+1}$, the $(n+1) \times(n+1)$ identity matrix.

Expanding the product of $U_{n} U_{n}^{-1}$ as we did in the proof of Theorem 4.4, proves this Theorem.
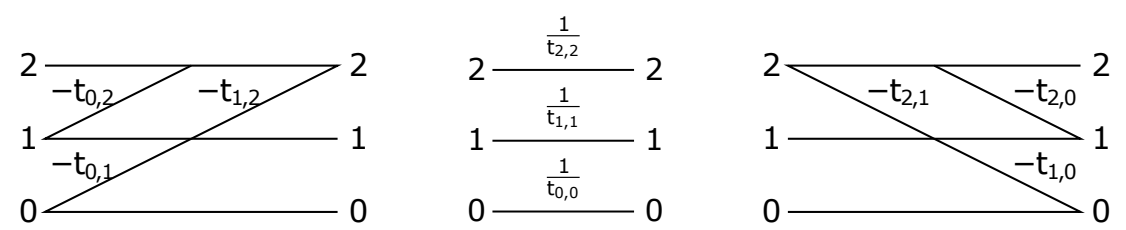

(a) $U$-type subnetwork of (b) $D$-type subnetwork of (c) $L$-type subnetwork of order 2 order 2 order 2

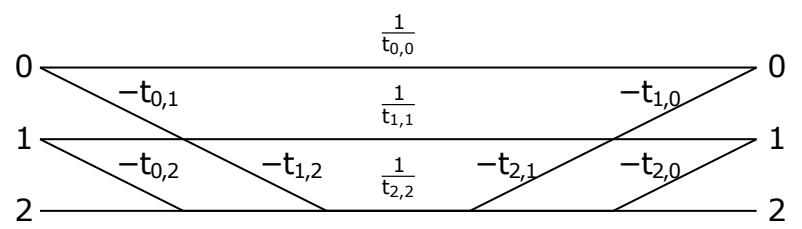

(d) Planar network of order 2 for the inverse matrix

FIGURE 6. Concatenation of essential planar subnetworks of order 2 to construct a network for the inverse of the weight matrix of the essential planar network presented in

Figure 4

Figure 6 illustrates an example of a planar network of order 2, corresponding to the inverse of a $3 \times 3$ totally positive matrix, obtained by concatenating three essential planar subnetworks of order 2. The order of the networks is reversed to account for the fact that $(L D U)^{-1}=U^{-1} D^{-1} L^{-1}$. The network 
is flipped horizontally (sources and sinks are numbered top-to-bottom from 0 to 2) to be closer to the essential planar network for better readability.

It is worth noting that all the results of this paper can be easily extended to invertible totally nonnegative matrices. This relies on a result due to Ando [1, Corollary 3.8]. This is equivalent to stating that the weights of the $D$-network, namely $t_{i, i}$, cannot be 0 for all $0 \leq i \leq n$; which guarantee the invertibility and, by Theorem 1.1 , the uniqueness of the $L U$-factorization with $L$ unit lower triangular. Therefore, it has a unique $L D U$-factorization with $L$ unit lower triangular, $D$ diagonal and $U$ unit upper triangular.

REMARK 4.8. The algorithm for finding the $L D U$-decomposition is divided into two parts. The first is extracting the $n^{2}$ essential weights from the original matrix. It turns out to be a reduced system of $n^{2}$ equations and has both time and space complexities of $\mathcal{O}\left(n^{2}\right)$. This algorithm is described in details in [5]. The second part is computing each entry of the matrices $L, D$ and $U$. Computation of the diagonal elements of $D$ is straightforward and has time complexity $\mathcal{O}(n)$. However, computing an off diagonal $(i, j)$-entry of $L$, or $U$, requires the generation of increasing sequences in $Q_{i, j}^{I}$. The number of these sequences for all $(i, j)$-entries is related to Fibonacci numbers which has an exponential rate of growth.

Finding the inverse, however, is divided into three steps; extracting the essential weights, calculating each entry of $L^{-1}, D^{-1}$ and $U^{-1}$, and multiplying the three matrices together. The first step is already discussed. The second is the same as discussed above for $L, D$ and $U$, but this time strictly decreasing sequences are considered and analogously, it has an exponential rate of growth. The third step has time complexity $\mathcal{O}\left(n^{3}\right)$.

Standard algorithms for finding the $L U$ factorization and the inverse rely on Gauss elimination and both have time complexity of $\mathcal{O}\left(n^{3}\right)$. Hence, standard algorithms are more efficient from a computational point of view. However, this note mainly focuses on a combinatorial description of the $L, D$, and $U$ factors of a totally positive matrix, and provides a combinatorial description of its inverse. In other words, we parametrize the $L D U$ factors and the inverse by the same parameters of the totally positive matrix.

\section{Numerical Examples}

In this section, we illustrate the Theorems presented in the previous sections via numerical examples. In the first example, we compute the inverse of $A$ using the combinatorial description of the inverses of the $L, D$ and $U$. introduced in Section 4. Consider the $4 \times 4$ matrix $A$ such that

$$
A=\left(\begin{array}{cccc}
3 & 6 & 18 & 306 \\
3 & 8 & 26 & 454 \\
6 & 22 & 77 & 1384 \\
60 & 262 & 958 & 17593
\end{array}\right)
$$


A direct computation of the minors verifies that $A$ is a totally positive matrix. Using the method described in [5], we obtain the following essential planar network corresponding to $A$ :

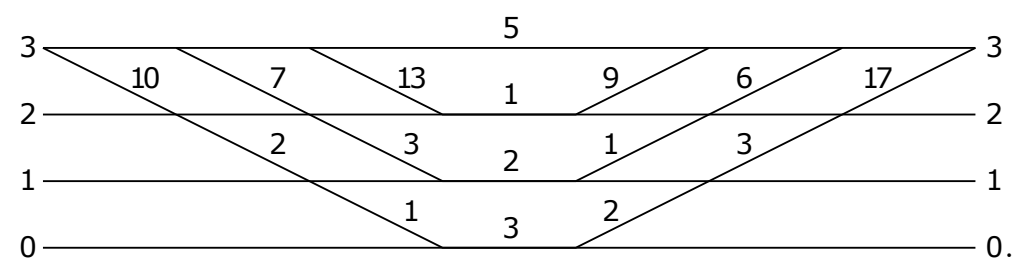

Using Theorem 2.12, we decompose this network into planar subnetworks to recover the $L D U$ factorization of $A$. The planar subnetworks and their corresponding weight matrices $L, D$ and $U$ are

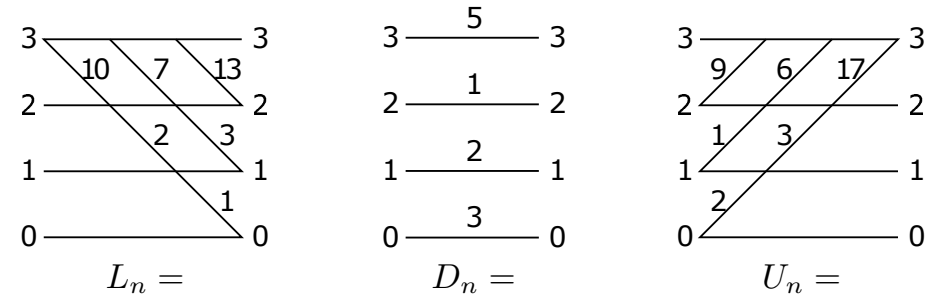

$$
\begin{aligned}
& \left(\begin{array}{cccc}
1 & 0 & 0 & 0 \\
1 & 1 & 0 & 0 \\
2 & 5 & 1 & 0 \\
20 & 71 & 30 & 1
\end{array}\right) \quad\left(\begin{array}{llll}
3 & 0 & 0 & 0 \\
0 & 2 & 0 & 0 \\
0 & 0 & 1 & 0 \\
0 & 0 & 0 & 5
\end{array}\right) \quad\left(\begin{array}{cccc}
1 & 2 & 6 & 102 \\
0 & 1 & 4 & 74 \\
0 & 0 & 1 & 32 \\
0 & 0 & 0 & 1
\end{array}\right) .
\end{aligned}
$$

Using the combinatorial interpretation of the inverses presented in Section 4, we can find the inverses of the $L, D$ and $U$ factors. The following is a combinatorial description of the inverses of the $L, D$ and $U$ factors.

$$
\begin{aligned}
& \begin{array}{l}
3 \\
\hline \\
\hline
\end{array} \\
& \begin{array}{l}
3 \frac{\frac{1}{5}}{2} 3 \\
1 \frac{1}{\frac{\frac{1}{2}}{2}} \text { 1 } \\
0 \frac{\frac{1}{3}}{D_{n}^{-1}=} 0
\end{array}
\end{aligned}
$$

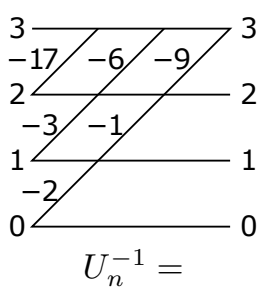

$$
\begin{aligned}
& \left(\begin{array}{cccc}
1 & 0 & 0 & 0 \\
-1 & 1 & 0 & 0 \\
3 & -5 & 1 & 0 \\
-39 & 79 & -30 & 1
\end{array}\right) \\
& \left(\begin{array}{cccc}
\frac{1}{3} & 0 & 0 & 0 \\
0 & \frac{1}{2} & 0 & 0 \\
0 & 0 & 1 & 0 \\
0 & 0 & 0 & \frac{1}{5}
\end{array}\right) \quad\left(\begin{array}{cccc}
1 & -2 & 2 & -18 \\
0 & 1 & -4 & 54 \\
0 & 0 & 1 & -32 \\
0 & 0 & 0 & 1
\end{array}\right)
\end{aligned}
$$


Since $\left(L_{n} D_{n} U_{n}\right)^{-1}=U_{n}^{-1} D_{n}^{-1} L_{n}^{-1}$, we reverse the order of the networks in the following way:

$$
\begin{aligned}
& \frac{U_{n}^{-1}=}{2} \\
& \begin{array}{l}
3 \frac{\frac{1}{5}}{3} 3 \\
2 \frac{1}{1 \frac{\frac{1}{2}}{2}} 1 \\
0 \frac{\frac{1}{3}}{D_{n}^{-1}=} 0
\end{array}
\end{aligned}
$$

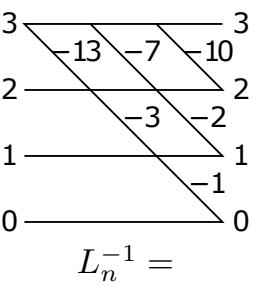

$$
\begin{aligned}
& \left(\begin{array}{cccc}
1 & -2 & 2 & -18 \\
0 & 1 & -4 & 54 \\
0 & 0 & 1 & -32 \\
0 & 0 & 0 & 1
\end{array}\right) \quad\left(\begin{array}{cccc}
\frac{1}{3} & 0 & 0 & 0 \\
0 & \frac{1}{2} & 0 & 0 \\
0 & 0 & 1 & 0 \\
0 & 0 & 0 & \frac{1}{5}
\end{array}\right) \quad\left(\begin{array}{cccc}
1 & 0 & 0 & 0 \\
-1 & 1 & 0 & 0 \\
3 & -5 & 1 & 0 \\
-39 & 79 & -30 & 1
\end{array}\right) .
\end{aligned}
$$

Finally, we concatenate all three subnetworks yields a planar network from which we recover the entries of the $A^{-1}$. For simplicity and conformity with the structure of all planar networks described in this note, we flip the concatenated network vertically, that is, we number the sources and sinks from top to bottom to obtain

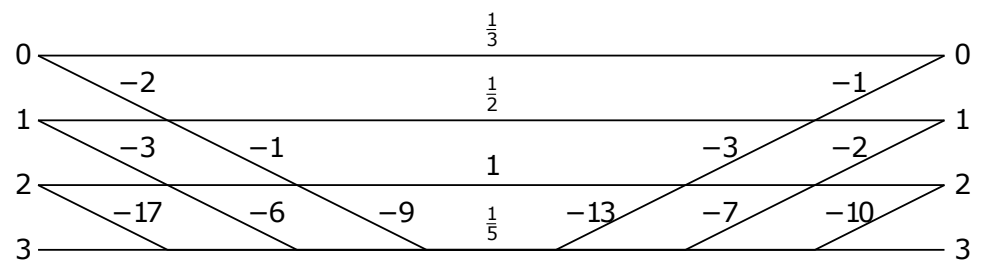

$$
A^{-1}=\left(\begin{array}{cccc}
\frac{2216}{15} & \frac{-1477}{5} & 110 & \frac{-18}{5} \\
\frac{-4337}{10} & \frac{8737}{10} & -328 & \frac{54}{5} \\
\frac{1263}{5} & \frac{-2553}{5} & 193 & \frac{-32}{5} \\
\frac{-39}{5} & \frac{79}{5} & -6 & \frac{1}{5}
\end{array}\right) .
$$

We can see from this example that there is a deterministic method for obtaining the weights of the inverse network from the essential weighting of the essential planar network. This method is best explained by the $L D U$ decomposition and inverses of the $L_{n}, D_{n}$, and $U_{n}$ factors.

In the second example, we compute the entries of the upper triangular $4 \times 4$ matrix $U_{3}$, using Proposition 3.2. The basis step is $U_{0}=[1]$. We know that,

$$
U_{1}^{-1}=\left(\begin{array}{cc}
1 & -t_{0,1} \\
0 & 1
\end{array}\right)
$$


Therefore, the $2 \times 2 F_{U_{1}}$ matrix is given by

$$
F_{U_{1}}=\left(\begin{array}{cc}
1 & t_{0,1} \\
0 & 1
\end{array}\right)
$$

Using the recursive formula introduced in Proposition 3.2, we obtain

$$
U_{1}=\left(U_{0} \oplus 1\right) F_{U_{1}}=\left(\begin{array}{cc}
1 & t_{0,1} \\
0 & 1
\end{array}\right) .
$$

Repeating the same process, we get $U_{2}^{-1}$,

$$
U_{2}^{-1}=\left(\begin{array}{ccc}
1 & -t_{0,1} & t_{0,1} t_{1,2} \\
0 & 1 & -\left(t_{0,2}+t_{1,2}\right) \\
0 & 0 & 1
\end{array}\right)
$$

Consequently, $F_{U_{2}}$ is the $3 \times 3$ matrix given by

$$
F_{U_{2}}=\left(\begin{array}{ccc}
1 & 0 & -t_{0,1} t_{1,2} \\
0 & 1 & t_{0,2}+t_{1,2} \\
0 & 0 & 1
\end{array}\right)
$$

Hence,

$$
U_{2}=\left(U_{1} \oplus 1\right) F_{U_{2}}=\left(\begin{array}{ccc}
1 & t_{0,1} & t_{0,1} t_{0,2} \\
0 & 1 & t_{0,2}+t_{1,2} \\
0 & 0 & 1
\end{array}\right)
$$

Again, we know that $U_{3}^{-1}$ is given by

$$
U_{3}^{-1}=\left(\begin{array}{cccc}
1 & -t_{0,1} & t_{0,1} t_{1,2} & -t_{0,1} t_{1,2} t_{2,3} \\
0 & 1 & -\left(t_{0,2}+t_{1,2}\right) & t_{0,2}\left(t_{1,3}+t_{2,3}\right)+t_{1,2} t_{2,3} \\
0 & 0 & 1 & -\left(t_{0,3}+t_{1,3}+t_{2,3}\right) \\
0 & 0 & 0 & 1
\end{array}\right)
$$

which means $F_{U_{3}}$ is given by

$$
F_{U_{3}}=\left(\begin{array}{cccc}
1 & 0 & 0 & t_{0,1} t_{1,2} t_{2,3} \\
0 & 1 & 0 & -t_{0,2}\left(t_{1,3}+t_{2,3}\right)-t_{1,2} t_{2,3} \\
0 & 0 & 1 & t_{0,3}+t_{1,3}+t_{2,3} \\
0 & 0 & 0 & 1
\end{array}\right)
$$

and finally

$$
\begin{aligned}
& U_{3}=\left(U_{2} \oplus 1\right) F_{U_{3}}= \\
& \left(\begin{array}{cccc}
1 & t_{0,1} & t_{0,1} t_{0,2} & t_{0,1} t_{0,2} t_{0,3} \\
0 & 1 & t_{0,2}+t_{1,2} & t_{1,2}\left(t_{0,3}+t_{1,3}\right)+t_{0,2} t_{0,3} \\
0 & 0 & 1 & t_{0,3}+t_{1,3}+t_{2,3} \\
0 & 0 & 0 & 1
\end{array}\right) .
\end{aligned}
$$




\section{REFERENCES}

[1] T. Ando, Totally positive matrices, Linear Algebra Appl. 90 (1987), 165-219.

[2] R. Cantó, P. Koev, B. Ricarte, and A. M. Urbano, LDU factorization of nonsingular totally nonpositive matrices, SIAM J. Matrix Anal. Appl. 167 (2008), 777-782.

[3] C. W. Cryer, The LU-factorization of totally positive matrices, Linear Algebra and Appl. 7 (1973), 83-92.

[4] S. M. Fallat and C. R. Johnson, Totally nonnegative matrices, Princeton University Press, Princeton, 2011.

[5] S. Fomin and A. Zelevinsky, Total positivity: tests and parametrizations, Math. Intelligencer 22 (2000), 23-33.

[6] F. R. Gantmacher and M. G. Krein, Sur les matrices complètement non négatives et oscillatoires, Compositio Math. 4 (1937), 445-476.

[7] F. R. Gantmacher, The theory of matrices, Chelsea, New York, 1959.

[8] M. Gasca and C. A. Micchelli, Eds., Total positivity and its applications, Mathematics and its Applications 359, Kluwer Academic Publishers, Dordrecht, The Netherlands, 1996.

[9] M. Gasca and J. M. Peña, On factorizations of totally positive matrices, in Total positivity and its applications, Kluwer Academic Publishers, Dordrecht, The Netherlands, 1996, 109-130.

[10] S. Karlin, Total positivity, Stanford University Press, Stanford, 1968.

\section{ElGebali}

Mathematics and Actuarial Science Department

The American University in Cairo

11853 Cairo

Egypt

E-mail: m.elgebali@aucegypt.edu

\section{N. El-Sissi}

Mathematics and Actuarial Science Department

The American University in Cairo

11853 Cairo

Egypt

E-mail: nelsissi@aucegypt.edu

Received: 12.12.2016.

Revised: 7.7.2017. 